\title{
EFECTO DE RECUBRIMIENTOS PROTECTORES SOBRE LA CALIDAD DEL MANGO (Mangifera indica L.) EN POSCOSECHA
}

\section{EFFECT OF PROTECTIVE COATINGS ON THE QUALITY OF MANGO (Mangifera indica L.) IN POSTHARVEST}

\author{
Eliana María Estrada Mesa ${ }^{1}$, Fabio Padilla Reyes ${ }^{2}$, Carlos Julio Márquez Cardozo ${ }^{3}$ \\ ${ }^{1}$ Ingeniera Biológica. Universidad Nacional de Colombia Facultad de Ciencias Agrarias. Calle 59A No 63-20 Medellín, Colom- \\ bia, e-mail: emestradam@unal.edu.co; ${ }^{2}$ Químico, Universidad Nacional de Colombia, e-mail: fapadillar@unal.edu.co; ${ }^{3} \mathrm{Ph} . \mathrm{D}$. \\ en Ciencias Agrarias, Profesor Asociado, Universidad Nacional de Colombia, e-mail: cjmarque@unal.edu.co
}

Rev. U.D.C.A Act. \& Div. Cient. 18(1): 181-188, Enero- Junio, 2015

\section{RESUMEN}

El mango (Mangifera indica L.) es un fruto climatérico de excelsas características sensoriales, pero presenta una vida útil o de anaquel muy corta, por lo tanto, es necesario explorar alternativas tecnológicas, que permitan prolongar su periodo poscosecha. Dentro de los desarrollos más estudiados, actualmente, se encuentra la aplicación de recubrimientos comestibles a frutas de interés comercial, que permitan mejorar sus características fisiológicas y sensoriales. En la presente investigación, se evaluó el efecto de dos recubrimientos comestibles sobre la calidad de frutos de mango. Los frutos fueron recubiertos con soluciones de almidón de yuca (Manihot esculenta C.) y pectina cítrica, en concentraciones de 1,5\%. Los mangos, se sumergieron en la solución durante dos minutos y se almacenaron a $11 \pm 1^{\circ} \mathrm{C}$ y $80 \pm 5 \%$ de HR. Se evaluó cada tres días, durante 12 días, la firmeza, la pérdida fisiológica de peso, los parámetros triestímulos de color; "CIELab", el índice de pardeamiento, los sólidos solubles totales, la acidez y la calidad sensorial. Se logró concluir que los recubrimientos comestibles, con base en almidón de yuca y pectina permitieron conservar los frutos de mango cv. Criollo durante 12 días almacenados, en refrigeración a $11 \pm 1^{\circ} \mathrm{C}$ y $80 \pm 5 \%$ de HR.

Palabras clave: Climatérico, hidrocoloides, características fisicoquímicas, almacenamiento.

\section{SUMMARY}

Mango (Mangifera indica L.) is a climacteric fruit of sublime sensory characteristics, but with a very short shelf life, so it is necessary to explore technological alternatives to extend its postharvest period. Within the improvements, most studied currently, the application of edible coatings for fruits of commercial interest that allow to improve their physiological and sensory characteristics, is found. In the present research the effect of two edible coatings on the quality of mango fruits was evaluated. The fruits were coated with solutions of cassava starch (Manihot esculenta C.) and citrus pectin at concentrations of $1.5 \%$; the mangoes were immersed in the solution for two minutes and stored at $11 \pm 1^{\circ} \mathrm{C}$ and $80 \pm 5 \%$ $\mathrm{RH}$. Firmness, physiological weight loss and the tristimulus color parameters; "CIELab", the browning index, total soluble solids, acidity and sensory quality was evaluated every three days during 12 days. It can be concluded that edible coatings based on cassava starch and pectin, allowed conserve mango fruits $\mathrm{cv}$. Criollo stored for 12 days at $11 \pm 1^{\circ} \mathrm{C}$ and $80 \pm 5 \% \mathrm{RH}$.

Key words: Climacteric, hydrocolloids, physicochemical characteristics, storage.

\section{INTRODUCCIÓN}

El mango (Mangifera indica L.) es uno de los frutos más apetecidos mundialmente para consumo en fresco, por su delicioso sabor y su alto valor nutritivo (Chiumarelli et al. 2011). Se ha reportado que es una importante fuente de ácido ascórbico, carotenoides y de compuestos fitoquímicos, entre ellos, los polifenoles, como galato de metilo, ácido gálico y ácido digálico y flavonoides, principalmente, quercetina y derivados de kaempferol, mangiferina e isómeros (Pierson et al. 2014). Por ser un fruto climatérico, la madurez organoléptica o de consumo la adquiere después de cosechado, entre los días seis y diez, en función de la variedad y del medio de almacenamiento. En este periodo presenta su principal pico respiratorio, que se identifica por el incremento de la actividad respiratoria, lo que desata la cascada de cambios en el color, el olor, el sabor y la textura (Pérez et al. 2004). 
El fruto es susceptible a enfermedades y sensible a daños por frío, cuando es almacenado a temperaturas menores de $13^{\circ} \mathrm{C}$, aspectos que lo hacen un producto de carácter perecedero, de vida útil relativamente corta, lo que limita su potencial de conservación, transporte, manipulación, almacenamiento y comercialización (Baldwin et al. 1999).

Frente a la alta perecibilidad del mango, se han implementado diferentes técnicas para contrarrestar esta situación y prolongar la vida poscosecha, entre ellas, se destacan: el almacenamiento en refrigeración, atmósferas modificadas y controladas, tratamientos hidrotérmicos, irradiación y formulaciones que contienen agentes biológicos, entre otros (Liu et al. 2014); todas ellas ejercen control en el deterioro de las características fisiológicas, sensoriales, fisicoquímicas y microbiológicas.

Otra opción consiste en la utilización de recubrimientos comestibles y su aplicación en los vegetales constituye una alternativa innovadora, para mantener la calidad de los frutos, ya que pueden actuar, favorablemente, sobre las características, fisiológicas, sensoriales, fisicoquímicas y microbiológicas, por lo tanto prolongan la vida útil de las frutas y mejoran su posibilidad de comercialización (Hoa \& Ducamp, 2008). Esta tecnología crea una atmósfera modificada alrededor del vegetal, proporcionando una barrera semipermeable a los gases implicados en el proceso respiratorio, el dióxido de carbono y el oxígeno y al vapor de agua, producto de la transpiración, debido a esta afectación fisiológica los vegetales retardan su metabolismo y la producción de etileno (Sothornvit \& Rodsamran, 2008).

La utilización de recubrimientos comestibles ha sido efectiva en algunas frutas, por ejemplo, recubrimiento a base de almidón de papa sobre guayaba (Sothornvit \& Rodsamran, 2008); en fresa recubierta con quitosan y almacenada a $10^{\circ} \mathrm{C}$ y $70 \pm 5 \%$ de $\mathrm{HR}$, se logró retrasar cambios en la pérdida de peso, firmeza y color externo, en comparación con las muestras no tratadas (Hernández-Muñoz et al. 2008); en níspero japonés, la utilización de soluciones de quitosan y de sucroéster de ácidos grasos demostró ser efectiva al reducir la pérdida fisiológica de peso, la tasa de respiración, la producción de etileno y en mantener la firmeza de los frutos (Márquez et al. 2009); en frutos de mango almacenados a $15^{\circ} \mathrm{C}$, se logró retrasar el cambio en el color, $\mathrm{pH}$ y acidez, mediante la aplicación de recubrimientos, con base en quitosan y polifenoles (Zambrano et al. 2011). También se han evaluado parámetros de calidad en rebanadas de mango pre-tratados con ácido cítrico y recubiertas con almidón de yuca o de alginato de sodio (Chiumarelli et al. 2011).

El objetivo fue determinar el efecto de dos recubrimientos comestibles a base de almidón de yuca (Manihot esculenta C.) y de pectina cítrica sobre la calidad en poscosecha del mango cv. Criolla almacenado a $11 \pm 1^{\circ} \mathrm{C}$ y $80 \pm 5 \%$ de HR durante 12 días.

\section{MATERIALES Y MÉTODOS}

Material vegetal: Frutos de mango cv. Criollo cosechados en zona rural del Municipio de Santa Bárbara, Antioquia (Colombia), en estado de madurez fisiológica fueron colectados y transportados a los Laboratorios de Frutas y Hortalizas y de Control de Calidad de Alimentos, de la Universidad Nacional de Colombia sede Medellín. Los mangos fueron seleccionados por apariencia externa, correspondiente a un fruto ovoide, aromático, de color de la cáscara número dos, según la clasificación para mangos criollos de azúcar; el calibre de los mangos utilizados fue 18 , con peso entre 161 y $200 \mathrm{~g}$. Se trabajó con un total de 108 unidades experimentales, divididas en tres grupos, correspondientes a los dos tratamientos y el control.

Formulación y desarrollo de los recubrimientos: Se prepararon dos recubrimientos comestibles en solución acuosa; en uno, como matriz principal, se utilizó almidón de yuca $(1,5 \%)$ $y$, en el otro, pectina cítrica $(1,5 \%)$, glicerol $(0,1 \%)$-como plastificante-, ácido ascórbico (2\%) -como antioxidante-, $\mathrm{CaCl}_{2}(0,1 \%)$-como mejorador de textura-, lecitina $(0,1 \%)$ -como emulsificante- y aceite de oliva $(0,5 \%)$-como agente barrera al vapor de agua-. La disolución, se realizó a $80^{\circ} \mathrm{C}$ con agitación continua durante cinco minutos (Márquez et al. 2009).

Aplicación de los recubrimientos: Los frutos fueron sumergidos en los recubrimientos durante dos minutos; los frutos control fueron inmersos en agua, durante el mismo tiempo. Después de la aplicación de los recubrimientos, los frutos se dejaron secar durante 120 minutos, bajo condiciones ambientales de laboratorio de $25^{\circ} \mathrm{C}$ y $65 \%$ de HR. Después del secado, se almacenaron a $11 \pm 1^{\circ} \mathrm{C}$ y $80 \pm 5 \%$ de HR (Hernández- Muñoz et al. 2008).

Cambios fisicoquímicos: La pérdida fisiológica de peso, se determinó gravimétricamente, con una balanza analítica y se expresó como porcentaje de pérdida de peso respecto al peso inicial, utilizando seis frutos por tratamiento, similar a lo aplicado por otros investigadores. Se extrajo un gramo de pulpa de cada uno de los seis mangos para cada tratamiento y el control, el cual, se utilizó para medir los sólidos solubles totales, usando un refractómetro óptico (Atago con escala de 0-32\%), método AOAC 932.12/90. La acidez titulable fue determinada empleando titulación acido-base con $\mathrm{NaOH}$ $0,1 \mathrm{~N}$ y expresada como porcentaje de ácido cítrico, método AOAC 942.05/90.

Índice de pardeamiento: El efecto de los recubrimientos comestibles sobre el índice de pardeamiento en mango criollo, 
se determinó mediante la medición de color, utilizando un espectrofotómetro de esfera con iluminante D65 y un observador de $10^{\circ}$, como referencia (modelo SP64, X-RITE Inc., MI, USA), la evaluación se realizó cada 3 días durante 12 días. Las lecturas se obtuvieron en las coordenadas de color CIE-La*b*, donde "L" corresponde a la luminosidad, con escala de 0 a 100, "a" se refiere al intervalo de colores entre el verde y el rojo, y "b" representa al intervalo de colores entre el azul y el amarillo. Se hicieron mediciones a seis unidades experimentales por cada tratamiento. El índice de pardeamiento (IP), se calculó mediante las ecuaciones 1 y 2 , que representa un indicador del deterioro externo del fruto (Maskan, 2001).

$$
\mathrm{IP}=\frac{[100(\mathrm{X}-0,31)]}{0,17}
$$

Ecuación 1

Donde:

$$
\mathrm{x}=\frac{(\mathrm{a} *+1,75 \mathrm{~L} *)}{(5,645 \mathrm{~L} *+\mathrm{a} *-3,012 \mathrm{~b} *)}
$$

Ecuación 2

Medida de la firmeza: La firmeza de los frutos de mango fue medida cada 3 días hasta los 12 días. Se determinó utilizando un texturómetro (TA-XT2i Stable Micro Systems), con celda de carga de $50 \mathrm{~kg}$, con una sonda cilíndrica de $2 \mathrm{~mm}$ de diámetro y se expresó como la fuerza máxima en Newton, necesaria para penetrar el fruto de mango (Hernández- Muñoz et al. 2008; Márquez et al. 2009).
Evaluación sensorial: La evaluación sensorial fue realizada para el día 12 de almacenamiento, debido a que después de este periodo de tiempo el fruto ya presentaba deterioro aparente, según los preensayos realizados; las pruebas sensoriales las ejecutaron nueve jueces conocedores de las características organolépticas del mango, mediante la valoración numérica de los atributos de color, de apariencia, de olor y de sabor. Las condiciones para la realización de las pruebas por parte de los jueces fueron a temperatura ambiente de laboratorio.

Análisis estadístico: Se utilizo un diseño experimental completamente al azar, con un arreglo factorial $3 \times 5$, donde 3 corresponde a los tratamientos y 5 a los periodos de evaluación en el tiempo, fueron utilizados seis unidades experimentales por tratamiento por periodo de evaluación; teniendo así 18 unidades experimentales por periodo, con 75 grados de libertad del error. Se aplicó análisis de varianza y la prueba de Tukey para la diferencia de medias.

\section{RESULTADOS Y DISCUSIÓN}

Cambios fisicoquímicos: La figura 1 muestra la tendencia en la pérdida fisiológica de peso durante el almacenamiento de los frutos, siendo mayor la pérdida en el control, con un 2,6 y $2,7 \%$, con respecto a los frutos recubiertos con base en almidón de yuca y pectina cítrica, respectivamente; no obstante, entre los recubrimientos no se encontró diferencia estadísticamente significativa al aplicar la prueba de comparación

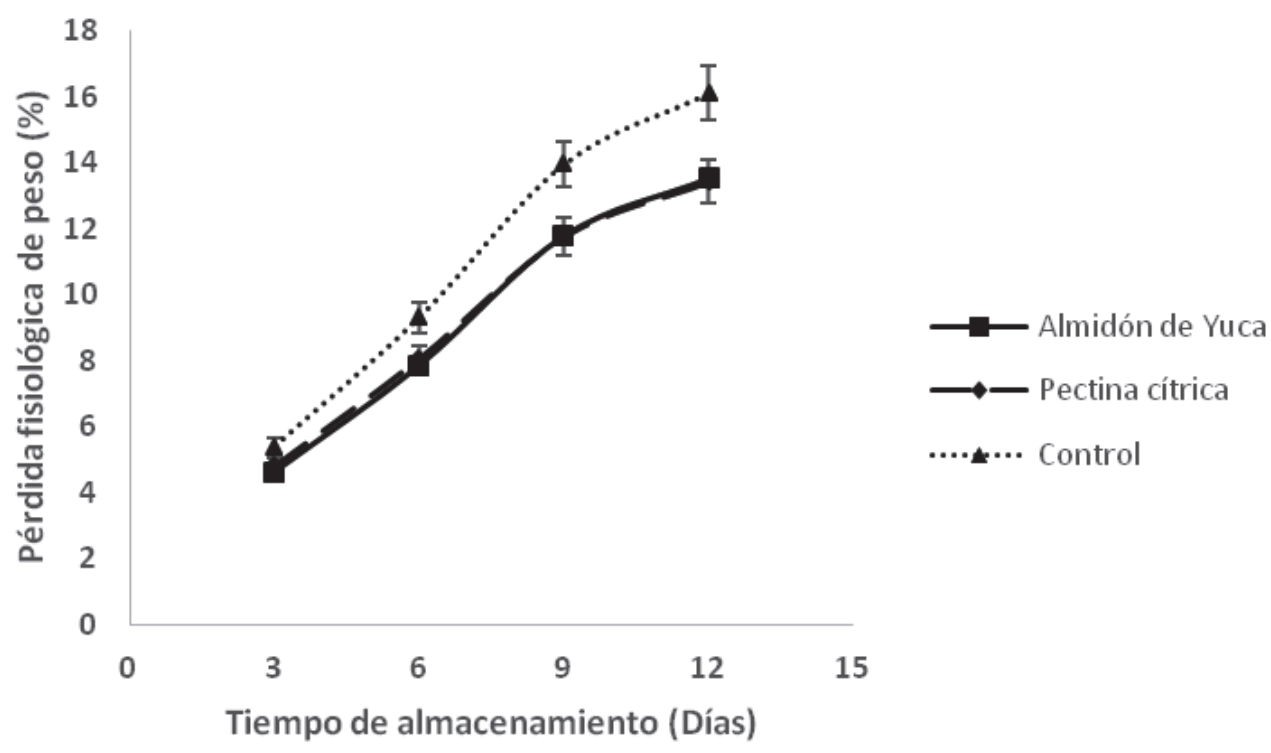

Figura 1. Pérdida fisiológica de peso en mango criollo (Mangifera indica L.), con recubrimientos comestibles, almacenado por 12 días, a $11 \pm 1^{\circ} \mathrm{C}$ y $80 \pm 5 \%$ de HR. Los símbolos representan la media para $n=6$ y las barras verticales los valores \pm del porcentaje de error al $5 \%$. 
de medias de Tukey ( $p>0,05)$. Se infiere, por lo tanto, que los recubrimientos comestibles proporcionaron una barrera protectora que restringió la transferencia de agua, es decir la transpiración y, de está manera, se retrasó la deshidratación de los frutos durante el almacenamiento, reduciendo la pérdida de peso; lo anterior está de acuerdo con lo reportado por otros investigadores, quienes encontraron que la aplicación de recubrimientos comestibles en mango inhibía la pérdida de peso (Valera et al. 2011).

La pérdida fisiológica de peso en los frutos se asocia, principalmente, con la transpiración y es propia de cada vegetal, la velocidad de la pérdida de agua depende del gradiente de presión entre el tejido de la fruta, la atmósfera circundante y la temperatura de almacenamiento. Diferencias de presión de vapor baja entre la fruta y sus ambiente externo y temperatura de refrigeración son las condiciones más recomendables para el almacenamiento de las frutas, entre ellas, el mango (Hernández- Muñoz et al. 2008).

La figura 2 presenta el comportamiento de los sólidos solubles totales (SST) expresados en grados Brix; el contenido de sólidos solubles totales se incrementó para todos los tratamientos hasta el sexto día de almacenamiento, resultados similares a los reportados por otros investigadores (Pérez et al. 2003; Santamaría et al. 2009). Para el día 12 de almace- namiento, se reportó que la mayor concentración de solidos solubles totales la presentaron los frutos con recubrimiento de almidón de yuca, correspondiente a 16,9 ${ }^{\circ}$ Brix mientras que los frutos con el recubrimiento de pectina cítrica y el control fueron de $13,51^{\circ}$ Brix y $13,01^{\circ}$ Brix, respectivamente. Se pudo establecer efecto significativo en el tiempo de almacenamiento al aplicar la prueba de comparación de medias de Tukey $(p<0,05)$. Este comportamiento corresponde a que los carbohidratos se incrementan durante el almacenamiento de las frutas, debido a la generación de sacarosa y otros carbohidratos de bajo peso molecular, a partir del almidón de reserva, con la participación de enzimas amilasas (Rosli et al. 2004).

La figura 3 muestra la acidez titulable, que decreció durante el periodo de almacenamiento, siendo menor para el día 12 , en los frutos sin recubrimiento, alcanzando valores de $0,48 \%$, mientras que se mantuvo en $0,79 \%$ y $0,61 \%$, en los frutos de mango recubiertos con almidón de yuca y pectina cítrica, respectivamente. Se pudo establecer efecto significativo en el tiempo de almacenamiento al aplicar la prueba de comparación de medias de Tukey $(\mathrm{p}<0,05)$; resultados similares fueron reportados por Chien et al. (2007). Lo anterior, probablemente, a que la atmósfera modificada generada por los recubrimientos acumula $\mathrm{CO}_{2}$ en el tejido y aumenta su acidez, además, que en los frutos control es probable que se

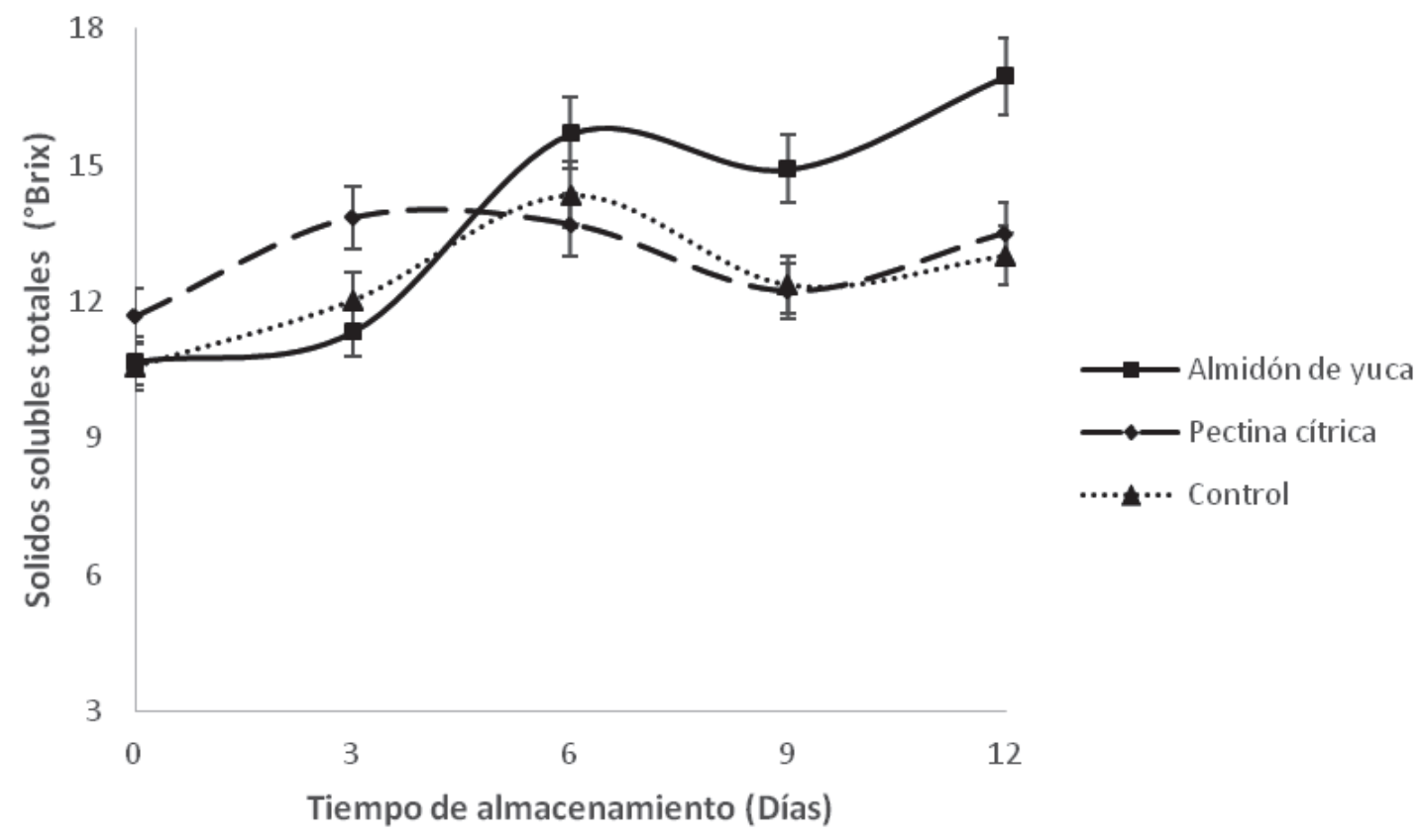

Figura 2. Comportamiento de los sólidos solubles totales (SST) en mango criollo (Mangifera indica L.), con recubrimientos comestibles, almacenado por 12 días, a $11 \pm 1^{\circ} \mathrm{C}$ y $80 \pm 5 \%$ de HR. Los símbolos representan la media para $\mathrm{n}=6$ y las barras verticales los valores \pm del porcentaje de error al $5 \%$. 


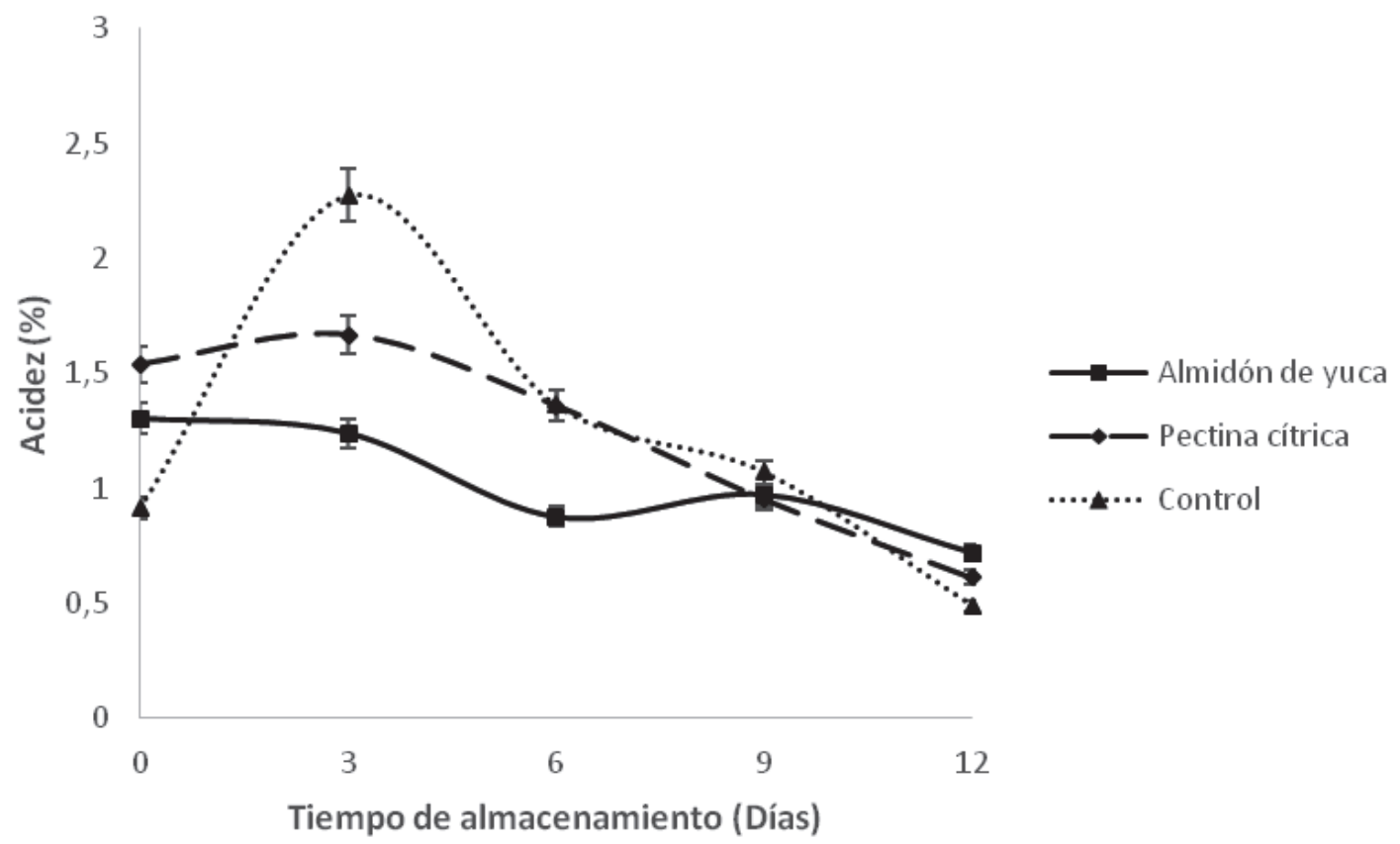

Figura 3. Comportamiento del porcentaje de acidez en mango criollo (Mangifera indica L.), con recubrimientos comestibles, almacenado por 12 días, a $11 \pm 1^{\circ} \mathrm{C}$ y $80 \pm 5 \%$ de HR. Los símbolos representan la media para $\mathrm{n}=6$ y las barras verticales los valores \pm del porcentaje de error al $5 \%$.

intensifiquen algunos procesos metabólicos, que consumen los ácidos presentes en el fruto (Zambrano et al. 2011).

La acidez tiende a disminuir durante el almacenamiento, hecho asociado con cambios metabólicos y la maduración de la fruta, que varía con la composición de los recubrimientos (Zambrano-Zaragoza et al. 2014). Otros investigadores han encontrado que la disminución del porcentaje de acidez puede estar asociada al incremento de la actividad enzimática en el ciclo del ácido cítrico (Pérez et al. 2003).

Determinación del índice de pardeamiento y medida de la firmeza: El índice de pardeamiento, se considera un parámetro necesario, tanto en procesos, donde se pueda llevar a cabo reacciones enzimáticas como no enzimáticas, (Zambrano-Zaragoza et al. 2014). Es una medida del contenido de pigmentos solubles producidos a partir de reacciones enzimáticas de pardeamiento (Kim et al. 2014). En la figura 4, se muestra el efecto de los tratamientos y del control en el índice de pardeamiento. No se encontró diferencia significativa entre los tratamientos ( $p>0,05)$; solamente hubo efecto significativo en el tiempo de almacenamiento $(p<0,05)$; sin embargo, se pudo establecer que el control presentó mayor incremento en el índice de pardeamiento con respecto a los recubrimientos, siendo de 131,6 mientras que para los frutos recubiertos con almidón de yuca y pectina cítrica, se encontraron valores de 128,2 y 116,0, respectivamente; lo anterior probablemente, debido a la modificación del oxígeno disponible y a cambios en la activación de la membrana celular, que modifican la actividad de la enzima polifenol oxidasa y su contacto con los sustratos (Zambrano-Zaragoza et al. 2014).

La textura es un atributo físico que se relaciona, de manera directa, con la firmeza del vegetal, aspecto crítico en la calidad de las frutas y hortalizas frescas y que se manifiesta en la aceptabilidad del consumidor (Hernández- Muñoz et al. 2008). La figura 5 muestra los cambios en la firmeza en función del tiempo de almacenamiento de los mangos recubiertos y el control; para la firmeza, no hubo interacción entre el tiempo y los tratamientos $(p>0,05)$, por lo tanto, se analizó el efecto simple del factor tiempo de almacenamiento, aplicando la prueba de comparación de medias de Tukey, que fue significativo $(\mathrm{p}<0,05)$; no obstante, la mayor disminución de la firmeza ocurrió en las muestras control, con una pérdida del 58\%, en comparación con el estado inicial. El recubrimiento, con base en almidón, presentó una pérdida de firmeza de $54 \%$, seguido por el de pectina cítrica, con una reducción de firmeza del 47\%. La firmeza está asociada a la degradación de las protopectinas insolubles a pectinas solubles, debido a los recubrimientos comestibles; esta si- 
tuación se puede ver retardada; sin embargo, en la presente investigación no se encontró diferencia estadísticamente significativa $(P>0,05)$, entre los tratamientos y el control, presentando decrecimiento continuo de la firmeza durante todo el periodo de evaluación (Valera et al. 2011).
Evaluación sensorial: Los frutos de mango recubiertos y el control, no presentaron diferencia estadísticamente significativa en la calidad sensorial total, evaluados a los 12 días de poscosecha, con un nivel de confianza del 95,0\%.

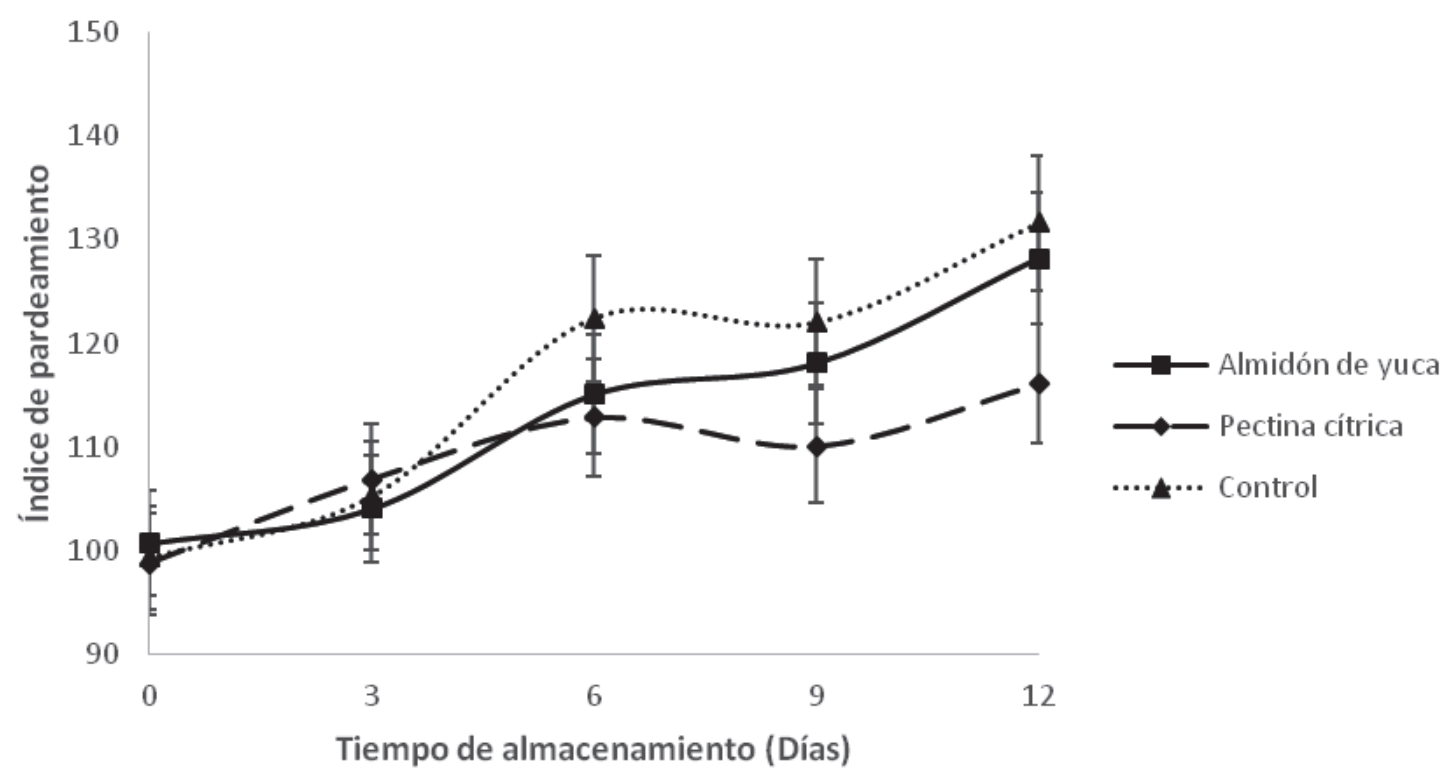

Figura 4. Índice de pardeamiento en mango criollo (Mangifera indica L.), con recubrimientos comestibles almacenado, por 12 días, a $11 \pm 1^{\circ} \mathrm{C}$ y $80 \pm 5 \%$ de HR. Los símbolos representan la media para $\mathrm{n}=6$ y las barras verticales los valores \pm del porcentaje de error al $5 \%$.

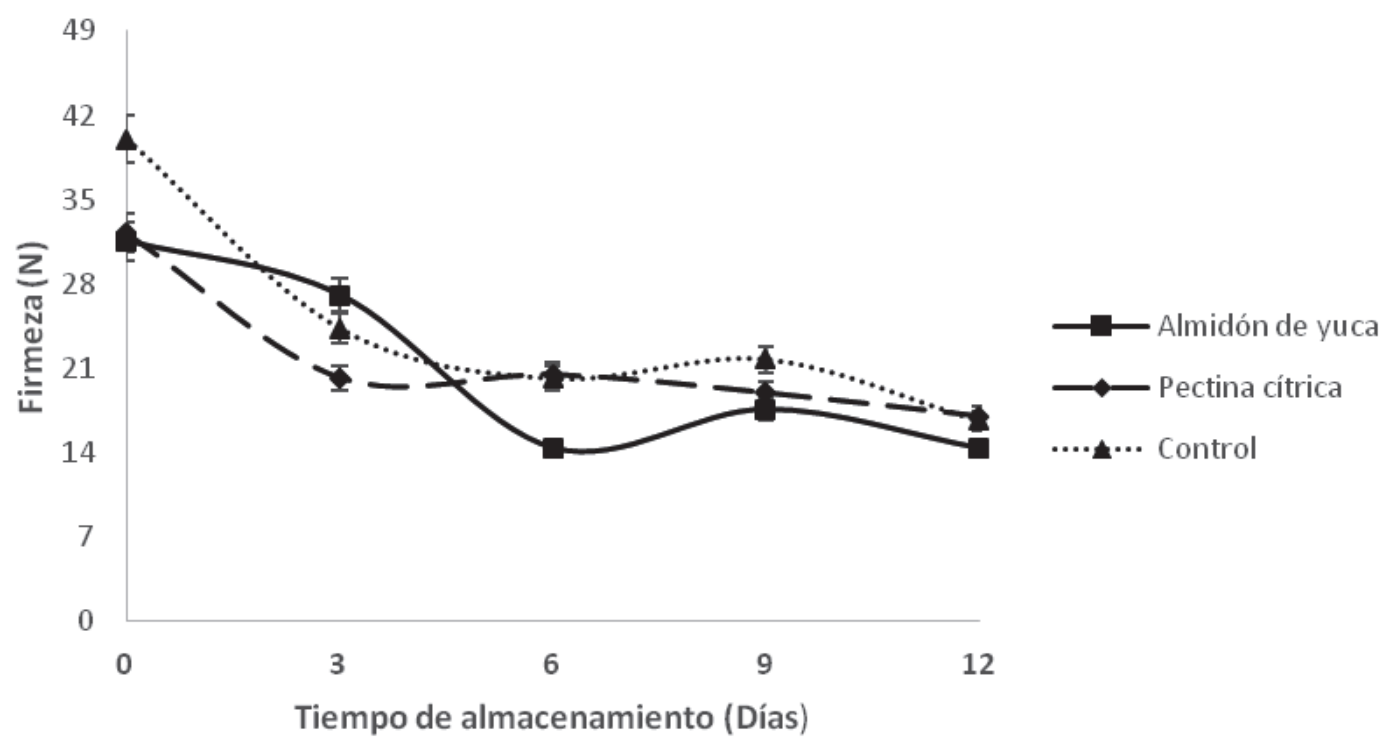

Figura 5. Firmeza en mango criollo (Mangifera indica L.), con recubrimientos comestibles, almacenado por 12 días, a $11 \pm 1^{\circ} \mathrm{C}$ y $80 \pm 5 \%$ de HR. Los símbolos representan la media para $\mathrm{n}=6$ y las barras verticales los valores \pm del porcentaje de error al $5 \%$. 
Se pudo concluir, de la presente investigación, que los recubrimientos comestibles con base en pectina cítrica y almidón de yuca, permitieron conservar los frutos de mango cv. Criollo durante 12 días, almacenados en refrigeración, a $11 \pm 1^{\circ} \mathrm{C}$ y $80 \pm 5 \%$ de HR.

Agradecimientos: Los autores agradecen al personal técnico de los laboratorios de Frutas y Hortalizas y de Control de Calidad de Alimentos de la Universidad Nacional de Colombia, sede Medellín. Conflicto de intereses: El manuscrito fue preparado y revisado con la participación de todos los autores, quienes declaramos que no existe conflicto de interés que ponga en riesgo la validez de los resultados presentados.

\section{BIBLIOGRAFÍA}

1. ASSOCIATION OF OFFICIAL ANALYTICAL CHEMISTS -AOAC-. 1970. Official methods of analysis of the Association of Official Analytical Chemists. $11^{\text {th }}$ ed. Washington. 1015p.

2. BALDWIN, E.A.; BURNS, J.K.; KAZOKAS, W. 1999. Effect of two edible coatings with different permeability characteristics on mango (Mangifera indica L.) ripening during storage. Postharv. Biol. Tec. 17:215226.

3. CHIEN, P.J.; SHEU, F.; YANG, F.H. 2007. Effects of edible chitosan coating on quality and shelf life of sliced mango fruit. J. Food Eng. 78(1):225-229.

4. CHIUMARELLI, M.; FERRARI, C.C.; SARANTÓPOULOS, C.; HUBINGER, M.D. 2011. Fresh cut 'Tommy Atkins' mango pre-treated with citric acid and coated with cassava (Manihot esculenta Crantz) starch or sodium alginate. Innov. Food Sci. Emerging Technol. 12:381-387.

5. HERNÁNDEZ-MUÑOZ, P.; ALMENAR, E.; VALLE, V.D.; VELEZ, D.; GAVARA, R. 2008. Effect of chitosan coating combined with postharvest calcium treatment on strawberry (Fragaria $\times$ ananassa) quality during refrigerated storage. Food Chem. 110(2):428-435.

6. HOA, T.T.; DUCAMP, M.N. 2008. Effects of different coatings on biochemical changes of 'cat Hoa loc' mangoes in storage. Postharv. Biol. Tec. 48:150-152.

7. KIM, D.H.; KIM, H.B.; CHUNG, H.S.; MOON, K.D. 2014. Browning control of fresh-cut lettuce by phytoncide treatment. Food Chem. 159:188-192.

8. LIU, K.; WANG, X.; YOUNG, M. 2014. Effect of bentonite/ potassium sorbate coatings on the quality of man- gos in storage at ambient temperature. J. Food Eng. 137:16-22.

9. MÁRQUEZ, C.J.; CARTAGENA, J.; PÉREZ-GAGO, M.B. 2009. Effect of edible coatings on japanese loquat (Eriobotrya japonica T.) postharvest quality. Vitae. (Colombia). 16(3):304-310.

10. MASKAN, M. 2001. Kinetics of color change of kiwifruits during hot air and microwave drying. J. Food Eng. 48(2):169-175.

11. PÉREZ, B.; BRINGAS, E.; CRUZ, L.; BÁEZ, R. 2003. Aplicación de cera comestible en mango. Parte I: Efecto en las características físico-químicas durante el almacenamiento comercial. Rev. Iberoam. Tec Postcosecha. 5(2):100-112.

12. PÉREZ, B.; BRINGAS, E.; MERCADO, J.N.; SAUCEDO, C.; CRUZ, L.; BÁEZ, R. 2004. Aplicación de cera comestible en mango. Parte II: estudios fisiológicos asociados a la maduración del fruto durante el almacenamiento comercial. Rev. Iberoam. Tec. Postcosecha. 6(1):24-33.

13. PIERSON, J.; MONTEITH, G.R.; ROBERTS-THOMSON, S.J.; DIETZGEN, R.G.; GIDLEY, M.J.; SHAW, P.N. 2014. Phytochemical extraction, characterisation and comparative distribution across four mango (Mangifera indica L.) fruit varieties. Food Chem. 149:253-263.

14. ROSLI, H. G.; CIVELLO, P.M.; MARTíNEZ, G.A. 2004. Changes in cell wall composition of three Fragaria $x$ ananassa cultivars with different softening rates during ripening. Plant Physiol. Biochem. 42(10):823831.

15. SANTAMARÍA, F.; DÍAZ, R.; SAURI, E.; ESPADAS, F.; SANTAMARÍA, J.M.; LARQUÉ, A. 2009. Características de calidad de frutos de papaya Maradol en la madurez de consumo. Agr. Téc. México. 35(3):347353.

16. SOTHORNVIT, R.; RODSAMRAN, P. 2008. Effect of a mango film on quality of whole and minimally processed mangoes. Postharvest Biol Tec. 47:407-415.

17. VALERA, A.; MATERANO, W.; MAFFEI, M.; QUINTERO, I.; ZAMBRANO, J. 2011. Uso de recubrimientos comestibles y baja temperatura para mantener la calidad de frutos de mango 'Bocado' durante el almacenamiento. Rev. Fac. Agron. (Venezuela). 28(1):600-608. 
18. ZAMBRANO, J.; MAFFEI, M.; MATERANO, W.; QUINTERO, I.; VALERA, A. 2011. Efecto de tres recubrimientos sobre algunos aspectos de calidad en mango 'Bocado' durante el almacenamiento. Rev. Fac. Agron. (Venezuela). 28(1):636-645.

19. ZAMBRANO-ZARAGOZA, M.L.; MERCADO-SILVA, E.; DEL REAL, A.; GUTIÉRREZ-CORTEZ, E.; CORNEJO-VILLEGAS, M.A.; QUINTANAR-GUERRERO, D.
2014. The effect of nano-coatings with $\alpha$-tocopherol and xanthan gum on shelf-life and browning index of fresh-cut "Red Delicious" apples. Inn. Food Sc. \& Emerging Tech. 22:188-196.

Recibido: Julio 24 de 2014

Aceptado: Febrero 25 de 2015

Cómo citar:

Estrada Mesa, E.M.; Padilla Reyes, F.; Márquez Cardozo, C.J. 2015. Efecto de recubrimientos protectores sobre la calidad del mango (Mangifera indica L.) en poscosecha. Rev. U.D.C.A Act. \& Div. Cient. 18(1): 181-188. 